\title{
Relevance of space anisotropy in the critical behavior of $m$-axial Lifshitz points
}

\author{
H. W. Diehl \\ Fachbereich Physik, Universität Duisburg-Essen, 45117 Essen, Germany \\ M. A. Shpot \\ Fachbereich Physik, Universität Duisburg-Essen, 45117 Essen, Germany \\ and Institute for Condensed Matter Physics, 79011 Lviv, Ukraine \\ R. K. P. Zia \\ Fachbereich Physik, Universität Duisburg-Essen, 45117 Essen, Germany \\ and Department of Physics, Virginia Polytechnic Institute and State University, Blacksburg, Virginia 24601, USA
}

(Received 3 July 2003; published 17 December 2003)

\begin{abstract}
The critical behavior of $d$-dimensional systems with $n$-component order parameter $\boldsymbol{\phi}$ is studied at an $m$-axial Lifshitz point where a wave-vector instability occurs in an $m$-dimensional subspace $\mathbb{R}^{m}(m>1)$. Field theoretic renormalization group techniques are exploited to examine the effects of terms in the Hamiltonian that break the rotational symmetry of the Euclidean group $\mathbb{E}(m)$. The framework for considering general operators of second order in $\boldsymbol{\phi}$ and fourth order in the derivatives $\partial_{\alpha}$ with respect to the Cartesian coordinates $x_{\alpha}$ of $\mathbb{R}^{m}$ is presented. For the specific case of systems with cubic anisotropy, the effects of having an additional term, $\sum_{\alpha=1}^{m}\left(\partial_{\alpha}^{2} \boldsymbol{\phi}\right)^{2}$, are investigated in an $\epsilon$ expansion about the upper critical dimension $d^{*}(m)=4+m / 2$. Its associated crossover exponent is computed to order $\epsilon^{2}$ and found to be positive, so that it is a relevant perturbation on a model isotropic in $\mathbb{R}^{m}$.
\end{abstract}

DOI: 10.1103/PhysRevB.68.224415 PACS number(s): 05.20.-y, 11.10.Kk, 64.60.Ak, 64.60.Fr

\section{INTRODUCTION}

According to the modern theory of critical phenomena, ${ }^{1,2}$ systems can be divided into universality classes such that the leading singularities of thermodynamic quantities of all members of a given class are the same. The key concept here is that the detailed differences between systems of any given class are "irrelevant." The most prominent and best studied universality classes are those of the $d$-dimensional $n$-vector models with short-range (ferromagnetic) interactions, conveniently represented by the standard $\phi^{4}$ model with Hamiltonian

$$
\mathcal{H}=\int d^{d} x\left[\frac{1}{2}(\nabla \boldsymbol{\phi})^{2}+\stackrel{\circ}{\tau} \frac{\stackrel{\circ}{2}}{\boldsymbol{\phi}^{2}}+\frac{\stackrel{\circ}{u}}{4 !}|\boldsymbol{\phi}|^{4}\right] .
$$

Here $\boldsymbol{\phi}=\left(\phi_{a}\right)$ (with $\left.a=1, \ldots, n\right)$ is an $n$-component orderparameter field, while $\stackrel{\circ}{\tau}$ and $\stackrel{\circ}{u}$ are the bare mass and coupling, respectively. To access a critical point, $\stackrel{\circ}{\tau}$ must be tuned (corresponding to tuning, say, the temperature of the system $T$ ) to a special value $\stackrel{\circ}{\tau}_{c}$ so that the renormalized $\tau$ (corresponding to the inverse susceptibility of the system) vanishes. The importance of this family of models derives from the fact that an enormous variety of experimentally studied systems belong to these universality classes. Specifically, irrelevant microscopic details include the lattice structure, the range of interactions (assumed finite and ferromagnetic) and pair interactions decaying with a sufficiently large power of the separation (e.g., van der Waals). ${ }^{3}$ Of course, not all microscopic details are irrelevant. Small admixtures of such interactions can be treated theoretically as "relevant pertur- bations" to the above universality classes, leading to predictions of experimentally measurable behavior of crossovers to other classes.

There are many extensions of the models (1), associated with systems with more complex types of microscopic interactions. For example, systems with competing interactions may display a richer variety of behavior. One particular simplified version is the axial next-nearest-neighbor Ising (ANNNI) model, ${ }^{4,5}$ in which an antiferromagnetic interaction between NNN pairs along one of the axes in a simple cubic lattice, in addition to the usual nearest-neighbor ferromagnetic interactions, is present. By tuning two (or more) control parameters in such systems, one can access a Lifshitz point. ${ }^{6}$

The focus of this paper is the critical properties and universality classes of $m$-axial Lifshitz points, ${ }^{4,6-9}$ possible in generalizations of the uniaxial $(m=1)$ ANNNI model. To describe these, we split the Euclidean space $R^{d}$ into $R^{m}$ $\times \mathbb{R}^{\bar{m}}$ with $\bar{m} \equiv d-m$. Let us label the coordinates in these subspaces as $x_{\alpha}, \alpha=1, \ldots, m$, and $x_{\beta}, \beta=m+1, \ldots, d$, respectively, and introduce the notations $\partial_{\alpha} \equiv \partial / \partial x_{\alpha}$ and $\partial_{\beta}$ $\equiv \partial / \partial x_{\beta}$. Then the Hamiltonian of these extended models reads

$$
\begin{aligned}
\mathcal{H}_{\text {iso }}= & \int d^{d} x\left[\frac{\circ}{\rho} \sum_{\alpha=1}^{m}\left(\partial_{\alpha} \boldsymbol{\phi}\right)^{2}+\frac{1}{2} \sum_{\beta=m+1}^{d}\left(\partial_{\beta} \boldsymbol{\phi}\right)^{2}\right. \\
& \left.+\frac{\stackrel{\circ}{\sigma_{1}}}{2}\left(\sum_{\alpha=1}^{m} \partial_{\alpha}^{2} \boldsymbol{\phi}\right)^{2}+\stackrel{\circ}{\tau} \boldsymbol{\phi}^{2}+\frac{\stackrel{\circ}{u}}{4 !}|\boldsymbol{\phi}|^{4}\right] .
\end{aligned}
$$

Provided the microscopic aspects [e.g., $d>d_{*}(m, n)$, the lower critical dimension here ${ }^{9}$ ] allow the system to be tuned to Lifshitz points, they occur at critical values $\stackrel{\circ}{\rho}_{\mathrm{LP}}$ and ${\stackrel{\circ}{\tau_{\mathrm{LP}}}}_{\text {of }}$ 
$\stackrel{\circ}{\rho}$ and $\stackrel{\circ}{\tau}$. Analogous to the above case, both of the renormalized parameters vanish at these points: $\rho=0$ and $\tau=0$. For $m=0$, the model (2) reduces to the standard isotropic $\phi^{4}$ theory of Eq. (1), with no Lifshitz points. At the other extreme, $m=d$, the system displays an isotropic Lifshitz point.

Although the model (2) was introduced more than 25 years ago, its systematic investigation beyond Landau theory via modern methods of field-theoretic renormalization group has just begun. ${ }^{10-15}$ Within the framework of an $\epsilon$ expansion $\left[\epsilon \equiv d^{*}(m)-d\right.$, where $d^{*}(m)$ is the upper critical dimension $4+m / 2$ and $0 \leqslant m \leqslant 8$ ], early studies were either restricted to special values of $m$ and a subset of critical exponents ${ }^{6,16,17}$ or else produced results ${ }^{18,17}$ to $O\left(\epsilon^{2}\right)$ in conflict with those of Sak and Grest ${ }^{16}$ and more recent work. ${ }^{10-15}$ Only recently has it become possible to master the enormous technical difficulties one encounters beyond the one-loop approximation. The full two-loop renormalization group (RG) analysis yielded results for all exponents (critical, crossover, and correction-to-scaling) to order $\epsilon^{2}$ for all values of $m \cdot{ }^{12-15} \mathrm{An}$ alternative picture of the Lifshitz point has been advocated by Leite. ${ }^{19}$ This has been critically assessed in Ref. 20 .

Let us also mention some earlier works on modifications of the model (2). Hornreich ${ }^{21}$ investigated the effects of contributions breaking the $O(n)$ invariance of the Hamiltonian, using a one-loop approximation. Folk and $\mathrm{Moser}^{22}$ studied Lifshitz points in systems with short-range and uniaxial dipolar interactions such as uniaxial ferroelectrics.

The purpose of the present paper is to examine the legitimacy of taking the fourth-order derivative terms of the Hamiltonian (2) as isotropic in the subspace $\mathbb{R}^{m}$. Made essentially for the sake of simplifying the computations, this assumption of " $m$-isotropy" is questionable, since the discrete lattice symmetries at microscopic scales are unlikely to respect full rotational invariance. Of course, we must account for these underlying symmetries at the continuum level when appropriate Hamiltonians are considered. Now, in the longwavelength limit, isotropy can be restored by appropriate rescaling of the axes at the level of second-order derivatives. However, there is no such luxury in general at the higher orders. Hence, the replacement

$$
\stackrel{\circ}{\sigma}_{1}\left(\sum_{\alpha=1}^{m} \partial_{\alpha}^{2} \boldsymbol{\phi}\right)^{2} \rightarrow \mathcal{T}_{\alpha_{1} \alpha_{2} \alpha_{3} \alpha_{4}}\left(\partial_{\alpha_{1}} \partial_{\alpha_{2}} \boldsymbol{\phi}\right) \partial_{\alpha_{3}} \partial_{\alpha_{4}} \boldsymbol{\phi}
$$

should be made in Eq. (2), where $\mathcal{T}$ is a linear combination

$$
\mathcal{T}_{\alpha_{1} \alpha_{2} \alpha_{3} \alpha_{4}}=\stackrel{\circ}{\sigma}_{i} T_{\alpha_{1} \alpha_{2} \alpha_{3} \alpha_{4}}^{(i)}
$$

of tensors $T^{(i)}$ compatible with the symmetry of the microscopic model considered. Here the summation convention is used: The doubly occurring index $i$ as well as all pairs of $\alpha$ indices are to be summed over. In general (the " $m$-clinic" case, a generalization of the familiar triclinic case for $m$ $=3$ ), there are

$$
n_{m}=\left(\begin{array}{c}
m+3 \\
4
\end{array}\right)
$$

such tensors. ${ }^{23,24}$ Instead of dealing with the $n_{m}$ coefficients $\stackrel{\circ}{\sigma}_{i}$, a convenient set are the $n_{m}-1$ dimensionless coupling constants

$$
\stackrel{\circ}{w}_{i}=\stackrel{\circ}{\sigma}_{i} / \stackrel{\circ}{\sigma}_{1}, \quad i=2, \ldots, n_{m}
$$

along with $\stackrel{\circ}{\sigma}_{1}$.

In this paper, we will focus our attention on a familiar example: the symmetry associated with an $m$-cube, i.e., cubic anisotropy. Besides the totally symmetric tensor

$$
S_{\alpha_{1} \alpha_{2} \alpha_{3} \alpha_{4}} \equiv \frac{1}{3}\left(\delta_{\alpha_{1} \alpha_{2}} \delta_{\alpha_{3} \alpha_{4}}+\delta_{\alpha_{1} \alpha_{4}} \delta_{\alpha_{3} \alpha_{2}}+\delta_{\alpha_{1} \alpha_{3}} \delta_{\alpha_{2} \alpha_{4}}\right),
$$

we have only another tensor, namely, the cubic

$$
\delta_{\alpha_{1} \alpha_{2} \alpha_{3} \alpha_{4}} \equiv \delta_{\alpha_{1} \alpha_{2}} \delta_{\alpha_{2} \alpha_{3}} \delta_{\alpha_{3} \alpha_{4}} .
$$

Thus Eq. (4) reduces to

$$
\mathcal{T}_{\alpha_{1} \alpha_{2} \alpha_{3} \alpha_{4}}=\stackrel{\circ}{\sigma}_{1} S_{\alpha_{1} \alpha_{2} \alpha_{3} \alpha_{4}}+\stackrel{\circ}{\sigma}_{2} \delta_{\alpha_{1} \alpha_{2} \alpha_{3} \alpha_{4}},
$$

and the Hamiltonian becomes

$$
\mathcal{H}=\mathcal{H}_{\text {iso }}+\frac{\stackrel{\circ}{\sigma}_{2}}{2} \int d^{d} x \sum_{\alpha=1}^{m}\left(\partial_{\alpha}^{2} \boldsymbol{\phi}\right)^{2} .
$$

Note that this model should represent the universality class of a simple generalization of the ANNNI model, i.e., from the uniaxial Ising $(m=n=1)$ to the $m$-axial $\mathrm{O}(n)$ case. Specifically, consider a simple cubic lattice $Z^{d}$ with classical $n$-vector spins $s_{i}$ of unit length on its sites $i$. Assume that the spins are coupled in an $\mathrm{O}(n)$ symmetric fashion, but with different characteristics within the two subspaces $\mathbb{R}^{m}$ and $\mathbb{R}^{\bar{m}}$. In the former, suppose the interactions are like those in the ANNNI model: nearest-neighbor ferromagnetic (of strength $J_{1}>0$ ) but second-neighbor antiferromagnetic (strength $J_{2}>0$ ) along each of the $m$ principal lattice directions. In the complementary subspace, let the interactions be only nearest-neighbor ferromagnetic (of strength $J_{3}>0$ ). The lattice Hamiltonian $\mathcal{H}_{\text {lat }}$ is explicitly

$$
\begin{aligned}
k_{\mathrm{B}} T \mathcal{H}_{\mathrm{lat}}= & -J_{1} \sum_{\substack{\langle i, j\rangle \\
i-j= \pm e_{\alpha}}} s_{i} \cdot s_{j} \\
& +J_{2} \sum_{\substack{\langle i, j\rangle \\
i-j= \pm 2 e_{\alpha}}} s_{i} \cdot s_{j}-J_{3} \sum_{\substack{\langle i, j\rangle \\
i-j= \pm e_{\beta}}} s_{i} \cdot s_{j} \cdot
\end{aligned}
$$

Denoting the Fourier transform of $s_{i}$ by $\tilde{\boldsymbol{s}}_{q}$, we recast this expression in Fourier space: $\left(q_{\alpha}, q_{\beta}\right)$. The first line yields a contribution $\propto \sum_{\alpha=1}^{m}\left[J_{2} \cos \left(2 q_{\alpha}\right)-J_{1} \cos \left(q_{\alpha}\right)\right]$ to the coefficient of $\left|\tilde{s}_{q}\right|^{2}$. The Lifshitz point can be accessed, at this naive level, by tuning the $O\left(q_{\alpha}^{2}\right)$ term to vanish, i.e., $J_{1}$ $=4 J_{2}$. Meanwhile, the $O\left(q_{\alpha}^{4}\right)$ term is precisely of the form of the $\stackrel{\circ}{\sigma}_{2}$ term in the coarse-grained Hamiltonian (10). Though this procedure does not directly yield a $\stackrel{\circ}{\sigma}_{1}$ term, there are two good reasons that such a term is unavoidable. 
Firstly, we generalized the ANNNI model in the simplest possible manner: All further-neighbor interactions couple only spins along the principal directions. ${ }^{25,26} \mathrm{Had}$ we introduced NNN bonds along diagonals (i.e., $\boldsymbol{i}-\boldsymbol{j}= \pm \boldsymbol{e}_{\alpha_{1}} \pm \boldsymbol{e}_{\alpha_{2}}$ ), there would be a contribution of the form $\sum_{\alpha_{1} \neq \alpha_{2}} q_{\alpha_{1}}^{2} q_{\alpha_{2}}^{2}\left|s_{q}\right|^{2}$, which involves both the symmetric tensor (7) as well as the cubic one (8). Second, the isotropic coupling will be automatically generated when shortwavelength degrees of freedom are integrated out, as will be shown in the RG analysis below. Thus, we expect that a wide class of lattice models similar to Eq. (11) will fall into the universality class described by the Hamiltonian (10).

Unless stated otherwise, we will restrict our attention, for simplicity, to this case [i.e., Eq. (9), tensor with cubic symmetry] and study only the Hamiltonian (10). Our goal is to examine the effects of this type of anisotropy on the isotropic $m$-axial Lifshitz point. Generalizing a two-loop RG analysis of the latter case, ${ }^{12,13}$ we will show that the cubic anisotropy $\propto \stackrel{\circ}{\sigma}_{2}$ is a relevant perturbation, at order $\epsilon^{2}$.

In the next section we present the formal framework for renormalization of the $m$-anisotropic model with general tensors of the form (4), including the associated RG equations. In Sec. II B, we specialize to the case (10) with only a cubic anisotropy. Since the anisotropy of interest appears in the momenta of a two-point vertex function, a two-loop computation is necessary. More explicit results, to first order in the cubic anisotropy, are provided, so that its effects on the RG flow near the isotropic fixed point, as well as scaling properties, can be investigated. For general values of $m$ and $n$, the $\epsilon$ expansion, to $O\left(\epsilon^{2}\right)$, of the associated crossover exponent, $\varphi_{2}(n, m, d)$, is obtained in terms of integrals over a single variable. In Sec. III, we compute these integrals, analytically for the special cases of $m=2,6$ and numerically for a range of other $m$ 's. An estimate of $\varphi_{2}(1,2,3)$ is presented. Concluding remarks are reserved for Sec. IV. Finally, there are three appendixes to which some details of our calculations have been relegated.

\section{RENORMALIZATION GROUP ANALYSIS}

\section{A. General anisotropy: Renormalization and RG equations}

To renormalize our theory with general " $m$-anisotropy" (4), we straightforwardly extend the considerations for the $m$-isotropic model (2) in Ref. 12. For the details in the analysis, we will follow the conventions and notations of Ref. 13. Here, we have $n_{m}$ variables, $\stackrel{\circ}{\sigma}_{i}$, whose scaling dimensions vanish at the Gaussian fixed point $\stackrel{\circ}{\rho}=\stackrel{\circ}{\tau}=\stackrel{\circ}{u}=0$. There are two consequences: Associated with each of the $\stackrel{\circ}{\sigma}_{i}$ is a renormalization factor $Z_{\sigma_{i}}$. Further, these quantities, as well as all other renormalization factors, become functions of $n_{m}$ dimensionless coupling constants, namely, the renormalized four-point coupling $u$ and $n_{m}-1$ renormalized counterparts of the bare variables $\stackrel{\circ}{w}_{i}$ [Eq. (6)]:

$$
w_{i} \equiv \sigma_{i} / \sigma_{1}, \quad i=2, \ldots, n_{m} .
$$

Accordingly, we reparametrize the theory as

$$
\begin{gathered}
\stackrel{\circ}{\sigma}_{i}=Z_{\sigma_{i}}(u, w) \sigma_{i}, \\
\boldsymbol{\phi}=\left[Z_{\phi}(u, w)\right]^{1 / 2} \boldsymbol{\phi}_{\mathrm{ren}}, \\
\left(\stackrel{\circ}{\rho}-\stackrel{\circ}{\rho}_{\mathrm{LP}}\right) \stackrel{\circ}{\sigma}_{1}^{-1 / 2}=\mu Z_{\rho}(u, \boldsymbol{w}) \rho, \\
\stackrel{\circ}{\tau}-\stackrel{\circ}{\mathrm{LP}}_{\mathrm{LP}}=\mu^{2} Z_{\tau}(u, \boldsymbol{w})\left[\tau+A_{\tau}(u, \boldsymbol{w}) \rho^{2}\right], \\
\stackrel{\circ}{\mathrm{u}} \stackrel{\circ}{1}_{1}^{-m / 4} F_{m, \epsilon}=\mu^{\epsilon} Z_{u}(u, \boldsymbol{w}) u,
\end{gathered}
$$

where $\mu$ is a momentum scale, $F_{m, \epsilon}$ denotes the normalization factor

$$
F_{m, \epsilon}=\frac{\Gamma(1+\epsilon / 2) \Gamma^{2}(1-\epsilon / 2) \Gamma(m / 4)}{(4 \pi)^{(8+m-2 \epsilon) / 4} \Gamma(2-\epsilon) \Gamma(m / 2)},
$$

and $\boldsymbol{w}$ stands for the set $\left\{w_{2}, \ldots, w_{n_{m}}\right\}$ of $n_{m}-1$ variables. Following Ref. 27, we have included a renormalization function $A_{\tau}(u, w)$ to absorb momentum-independent poles proportional $\rho^{2}$ of the two-point vertex function.

The fact that the theory must reduce for $\boldsymbol{w}=\mathbf{0}$ to the $m$-isotropic one implies the relations

$$
\begin{gathered}
Z_{\iota}(u, \boldsymbol{w}=\mathbf{0})=Z_{\iota}^{\mathrm{SD}}(u), \quad \iota=\phi, u, \tau, \rho \\
Z_{\sigma_{1}}(u, \boldsymbol{w}=\mathbf{0})=Z_{\sigma}^{\mathrm{SD}}(u),
\end{gathered}
$$

where the $Z$ factors marked by the superscript SD are those of Ref. 13. The function $A_{\tau}(u, \boldsymbol{w}=\mathbf{0})$ has been computed to one-loop order in Ref. 27. Its explicit form will not be needed in the following.

Turning to the RG equations, we use the notation $\left.\partial_{\mu}\right|_{0}$ for $\mu$ derivatives at fixed bare variables $\left(\stackrel{\circ}{u}, \stackrel{\circ}{\sigma}_{i}, \stackrel{\circ}{\tau}\right.$, and $\left.\stackrel{\circ}{\rho}\right)$, and define the $\beta$ and exponent functions

$$
\begin{gathered}
\left.\beta_{\kappa} \equiv \mu \partial_{\mu}\right|_{0} \kappa, \quad \kappa=u, \tau, \rho, \sigma_{i}, \\
\left.\eta_{\lambda}(u, w) \equiv \mu \partial_{\mu}\right|_{0} \ln Z_{\lambda}, \quad \lambda=\phi, u, \tau, \rho, \sigma_{i} .
\end{gathered}
$$

The functions $\eta_{\lambda}$ depend only on $u$ and $\boldsymbol{w}$. Since we use minimal subtraction of poles, they are even independent of $\epsilon$. In terms of these variables and ${ }^{27}$

$$
b_{\tau}(u, w) \equiv A_{\tau}\left[\left.\mu \partial_{\mu}\right|_{0} \ln A_{\tau}+\eta_{\tau}-2 \eta_{\rho}\right],
$$

the $\beta$ functions can be written as

$$
\begin{gathered}
\beta_{u}=-\left[\epsilon+\eta_{u}(u, \boldsymbol{w})\right] u, \\
\beta_{\tau}=-\left[2+\eta_{\tau}(u, \boldsymbol{w})\right] \tau-\rho^{2} b_{\tau}(u, \boldsymbol{w}), \\
\beta_{\rho}=-\left[1+\eta_{\rho}(u, \boldsymbol{w})\right] \rho, \\
\beta_{\sigma_{i}}=-\eta_{\sigma_{i}}(u, \boldsymbol{w}) \sigma_{i} .
\end{gathered}
$$

Also, since we fixed all renormalization factors $Z_{\lambda}$ such that the regular part of their Laurent series in $\epsilon$ is exactly unity, the associated $\eta_{\lambda}$ functions are related to the residues of the $Z_{\lambda}$ 's via

$$
\eta_{\lambda}=-u \partial_{u} \operatorname{Res}_{\epsilon=0} Z_{\lambda}
$$


In terms of the operator

$$
\mathcal{D}_{\mu} \equiv \mu \partial_{\mu}+\sum_{\kappa} \beta_{\kappa} \partial_{\kappa}
$$

the RG equations of the renormalized $N$-point cumulant functions $G_{\mathrm{ren}}^{(N)}(\boldsymbol{x}) \equiv\left\langle\Pi_{j=1}^{N} \phi_{a_{j}, \text { ren }}\left(\boldsymbol{x}_{j}\right)\right\rangle^{\text {cum }}$ and the corresponding vertex functions $\Gamma_{\text {ren }}^{(N)}$ read, respectively,

$$
\left[\mathcal{D}_{\mu}+\frac{N}{2} \eta_{\phi}\right] G_{\text {ren }}^{(N)}=0, \quad\left[\mathcal{D}_{\mu}-\frac{N}{2} \eta_{\phi}\right] \Gamma_{\text {ren }}^{(N)}=0 .
$$

Being dimensionless, $\boldsymbol{w}$ will appear in the Z's to arbitrary orders in general, even though we are dealing with the systematics of an expansion in powers of $u$ (the loop expansion). However, our principal goal here is a local stability analysis of the model (10) about the isotropic fixed point

$$
\mathcal{P}_{\text {iso }}^{*}:\left(u^{*}, \boldsymbol{w}=\mathbf{0}\right)
$$

where $u^{*}$ is the nontrivial zero of $\beta_{u}(\epsilon, u, \boldsymbol{w}=\mathbf{0})$ the explicit form of which, up to $O\left(\epsilon^{2}\right)$, is given in Eq. (60) of Ref. 13. To this end, we can linearize about $\mathcal{P}_{\text {iso }}^{*}$. Hence it will be sufficient to compute the counterterms to first order in $\boldsymbol{w}$.

\section{B. Cubic anisotropy: RG flow and scaling}

Given the general framework above, we turn to explicit results for the particular case with only a cubic anisotropy. With just $\sigma_{1}$ and $\sigma_{2}$, we have only one $w$ (and no need for the set $\boldsymbol{w})$. Furthermore, we need only terms linear in $w$. Referring the reader to Appendix A for the computational details, we note here that the pole terms of the two-loop graph $\bigodot$ yield the renormalization factors

$$
\begin{aligned}
Z_{\phi}= & 1-\frac{n+2}{3} \frac{1}{12(8-m)}\left[j_{\phi}(m)-36 i_{\phi}(m) w\right] \frac{u^{2}}{\epsilon} \\
& +O\left(w^{2}, u^{3}\right), \\
Z_{\phi} Z_{\sigma_{1}}= & 1+\frac{n+2}{3} \frac{1}{96 m(m+2)}\left[j_{\sigma}(m)-36 i_{\sigma_{1}}(m) w\right] \frac{u^{2}}{\epsilon} \\
& +O\left(w^{2}, u^{3}\right),
\end{aligned}
$$

and

$$
\begin{aligned}
Z_{\phi} Z_{\sigma_{2}}= & 1-\frac{n+2}{3} \frac{4 i_{\sigma_{2}}(m)}{m(m+2)(m+4)(m+6)} \frac{u^{2}}{\epsilon} \\
& +O\left(w u^{2}, u^{3}\right) .
\end{aligned}
$$

Here, $j_{\phi}(m)$ and $j_{\sigma}(m)$ are single-variable integrals encountered in Ref. 13. Their definitions are noted for the readers' convenience in Appendix A: Eqs. (A28) and (A29). Though more complicated, $i_{\phi}(m), i_{\sigma_{1}}(m)$, and $i_{\sigma_{2}}(m)$ are analogous integrals, defined in Eqs. (A24)-(A26).

From the structure of these $Z$ factors, it is clear that, at this two-loop order, both $\eta_{\sigma_{1}}$ and $\eta_{\sigma_{2}}$ are proportional to [ $n$ $+2) / 3] u^{2}$ with coefficients linear in $w$. As a result of Eq. (27), and keeping only terms to first order in $\sigma_{2}$, we find the associated $\beta$ functions to be of the form

$$
\left(\begin{array}{l}
\beta_{\sigma_{1}} \\
\beta_{\sigma_{2}}
\end{array}\right)=-2 \frac{n+2}{3} u^{2}\left(\begin{array}{cc}
K_{11} & K_{12} \\
0 & K_{22}
\end{array}\right)\left(\begin{array}{l}
\sigma_{1} \\
\sigma_{2}
\end{array}\right)[1+O(w)],
$$

where

$$
\begin{gathered}
K_{11} \equiv \frac{j_{\phi}(m)}{12(8-m)}+\frac{j_{\sigma}(m)}{96 m(m+2)}, \\
K_{12} \equiv-\frac{3 i_{\phi}(m)}{8-m}-\frac{3 i_{\sigma_{1}}(m)}{8 m(m+2)}, \\
K_{22} \equiv \frac{j_{\phi}(m)}{12(8-m)}-\frac{4 i_{\sigma_{2}}(m)}{m(m+2)(m+4)(m+6)},
\end{gathered}
$$

are constants, independent of the couplings.

Since our main interest is the neighborhood of $\mathcal{P}_{\text {iso }}^{*}$, we need to evaluate $u$ in this equation only at the $m$-isotropic fixed point,

$$
u^{*}=\frac{6 \epsilon}{n+8}+O\left(\epsilon^{2}\right)
$$

Given the form of the matrix in Eq. (35), the eigenvalues are trivially obtained, the first of which is just $\eta_{\sigma}^{*}$ in Refs. 12 and 13:

$$
\begin{gathered}
\eta_{\sigma_{1}}^{*} \equiv \eta_{\sigma}^{*}=-\frac{24(n+2)}{(n+8)^{2}} K_{11} \epsilon^{2}+O\left(\epsilon^{3}\right), \\
\eta_{\sigma_{2}}^{*} \equiv-\frac{24(n+2)}{(n+8)^{2}} K_{22} \epsilon^{2}+O\left(\epsilon^{3}\right) .
\end{gathered}
$$

Associated with these are, respectively, the (linear) scaling fields:

$$
\sigma_{1}+b \sigma_{2} \text { and } \sigma_{2},
$$

where $b \equiv K_{12} / K_{11}-K_{22}$. Near $\mathcal{P}_{\text {iso }}^{*}$, we may drop the irrelevant contributions proportional to $u-u^{*}$, so that the flow equations

$$
\ell \frac{\partial}{d \ell} \bar{\sigma}_{i}=\beta_{\sigma_{i}}
$$

are solved by $\bar{\sigma}_{i}(\ell)$. Imposing the initial conditions $\bar{\sigma}_{i}(1)$ $=\sigma_{i}$, these take the asymptotic forms 


$$
\begin{gathered}
\bar{\sigma}_{1}(\ell) \approx\left(\sigma_{1}+b \sigma_{2}\right) \ell^{-\eta_{\sigma}^{*}-b \sigma_{2} \ell^{-} \eta_{\sigma_{2}}^{*}} \\
\bar{\sigma}_{2}(\ell) \approx \sigma_{2} \ell^{-\eta_{\sigma_{2}}^{*}}
\end{gathered}
$$

Thus, to the order of interest, the anisotropy $w$ leads to a dependence on the following ratio of running variables:

$$
\begin{gathered}
\bar{\sigma}_{2}(\ell) / \bar{\sigma}_{1}(\ell) \approx\left(\sigma_{2} / \sigma_{1}\right) \ell^{-\left(\eta_{\sigma_{2}}^{*}-\eta_{\sigma}^{*}\right)} \\
=\left[w+O\left(w^{2}\right)\right] \ell^{-\left(\eta_{\sigma_{2}}^{*}-\eta_{\sigma}^{*}\right)}
\end{gathered}
$$

As we will show, $\eta_{\sigma_{2}}^{*}-\eta_{\sigma}^{*}$ is positive, so that the effect of $w$ is more significant in the infrared limit $(\ell \rightarrow 0)$. Therefore, we introduce the anisotropy crossover exponent

$$
\varphi_{2} \equiv \nu_{l 2}\left(\eta_{\sigma_{2}}^{*}-\eta_{\sigma}^{*}\right)
$$

which governs the scaling behavior of $w$ with $\tau: w \sim \tau^{-\varphi_{2}}$. Since the $\eta$ 's are already of $O\left(\epsilon^{2}\right)$, we may insert the zerothorder value for $\nu_{l 2}$ (i.e., 1/2) to obtain

$$
\begin{aligned}
\varphi_{2}= & \frac{n+2}{(n+8)^{2}} \frac{1}{m(m+2)}\left[\frac{48 i_{\sigma_{2}}(m)}{(m+4)(m+6)}+\frac{j_{\sigma}(m)}{8}\right] \epsilon^{2} \\
& +O\left(\epsilon^{3}\right) .
\end{aligned}
$$

As a consequence of the contribution proportional to $\rho^{2}$ of $\beta_{\tau}$ [see Eq. (25)], the variable $\tau$ is not a scaling field. Proceeding similarly as in Ref. 27, we can define a nonlinear scaling field ${ }^{28}$

$$
g_{\tau}=\tau+c_{\rho^{2}}^{\tau}(u) \rho^{2}+c_{\rho^{2}, w}^{\tau}(u) w \rho^{2}+\cdots
$$

with the asymptotic scale dependence $\bar{g}_{\tau}(\ell) \sim l^{-1 / \nu_{l 2}} g_{\tau}$, where the ellipsis stands for terms of higher order in $w$.

Utilizing the above results, one can generalize the considerations of Refs. 12 and27 in a straightforward fashion to obtain the scaling forms of the renormalized $\mathrm{N}$-point cumulants $G^{(N)}$

$$
\begin{aligned}
G^{(N)} & {\left[\left\{x_{\alpha}, x_{\beta}\right\} ; g_{\tau}, \rho, \sigma_{1}, \sigma_{2}, u, \mu\right] } \\
& \approx g_{\tau}^{-\nu_{l 2} \Delta_{G}} \Upsilon_{G}\left[\left\{\frac{\sqrt{\mu} x_{\alpha}}{\sigma^{1 / 4} g_{\tau}^{\nu_{l 4}}}, \frac{\mu x_{\beta}}{g_{\tau}^{\nu_{l 2}}}\right\} ; \frac{\rho}{g_{\tau}^{\varphi}}, \frac{w}{g_{\tau}^{\varphi_{2}}}\right],
\end{aligned}
$$

where $\Delta_{G}$ is the scaling dimension of $G^{(N)}$ :

$$
\Delta_{G}=(N / 2)\left[d-2+\eta_{l 2}+m(\theta-1)\right] .
$$

With the exception of $\varphi_{2}$, explicit expressions for all exponents, up to $O\left(\epsilon^{2}\right)$, are given in Refs. 12 and 13. In the next section, we present a brief summary of the steps for computing $\varphi_{2}$.

\section{EXPLICIT RESULTS FOR THE ANISOTROPY CROSSOVER EXPONENT}

From Eq. (44), we see that only two integrals are needed for finding the exponent $\varphi_{2}$, namely, $j_{\sigma}(m)$ and $i_{\sigma_{2}}(m)$. As shown in Appendix A, all integrations can be performed ana-
TABLE I. Numerical values of the integrals $j_{\sigma}(m), i_{\sigma_{2}}(m)$, and the coefficients $C(m)$ of $\varphi_{2}$ introduced in Eq. (53).

\begin{tabular}{lccc}
\hline \hline$m$ & $j_{\sigma}(m)$ & $i_{\sigma_{2}}(m)$ & $C(m)$ \\
\hline 2 & 4.74074 & 0.074074 & 0.00309 \\
3 & 10.804 & 0.24682 & 0.00380 \\
4 & 20.067 & 0.6175 & 0.00444 \\
5 & 32.95 & 1.279 & 0.00501 \\
6 & 49.7778 & 2.325428 & 0.00552 \\
\hline \hline
\end{tabular}

lytically, except the one over the scaling variable

$$
v \equiv \frac{\sqrt{x_{\alpha} x_{\alpha}}}{\left(x_{\beta} x_{\beta}\right)^{1 / 4}}
$$

for which we resort to numerical means. The first, $j_{\sigma}$, is familiar from Ref. 13, recalled here in Table I.

The second involves, in general, a product of three hypergeometric functions. As outlined in Appendix E of Ref. 13, we write

$$
i_{\sigma_{2}}(m)=\int_{0}^{\infty} d v I_{m}(v)=\int_{0}^{v_{0}} d v I_{m}(v)+R_{m}\left(v_{0}\right)
$$

splitting the integral into a contribution from a finite interval $\left(0, v_{0}\right)$ and a remainder $R_{m}\left(v_{0}\right)$. The point $v_{0}$ was chosen so that standard numerical integration routines (specifically, MATHEMATICA $^{29}$ ) yield sufficiently accurate results for the first term and a few terms of the asymptotic expansion for $I_{m}$ suffice for evaluating $R_{m}$ approximately. In practice, we chose $v_{0} \simeq 9.5$ and just the leading term of the asymptotic expansion. The latter can be computed analytically and leads to

$$
R_{m}\left(v_{0}\right) \approx \frac{3 \sqrt{\pi} 2^{8-m}(m-2)^{2}}{\Gamma(m / 2) \Gamma\left(\frac{1}{2}+m / 4\right) \Gamma(2-m / 4)} \frac{v_{0}^{m-8}}{8-m} .
$$

Combining this with the numerical integration over $\left(0, v_{0}\right)$, we arrive at the values of $i_{\sigma_{2}}(m)$ displayed in Table I. ${ }^{30}$

As discussed in Ref. 13, the cases $m=2$ and $m=6$ are quite special. The scaling functions from which $I_{m}(v)$ is formed-and hence $I_{m}(v)$ itself-reduce to elementary functions. [From another perspective, their asymptotic expansions terminate at low orders: ${ }^{12,13}$ See Eqs. (C1), (C7) and (B8), (B9). Indeed, the approximation (50) even vanishes for $m=2$.] As a result, $i_{\sigma_{2}}$ can be computed analytically. The results (see Appendix $\mathrm{C}$ ),

$$
i_{\sigma_{2}}(2)=\frac{2}{27}, \quad i_{\sigma_{2}}(6)=20\left(8 \ln \frac{4}{3}-\frac{59}{27}\right),
$$

are in conformity with the values quoted in Table I and provide useful checks of our numerical procedure. For completeness, let us also recall the analytic values

$$
j_{\sigma}(2)=\frac{128}{27}, \quad j_{\sigma}(6)=\frac{448}{9}
$$


from Ref. 13.

Inserting the values from Table I into Eq. (44), we write the anisotropy crossover exponent as

$$
\varphi_{2}=\frac{27(n+2)}{(n+8)^{2}} C(m) \epsilon^{2}+O\left(\epsilon^{3}\right) .
$$

Here the $C(m)$ are the $\epsilon^{2}$ coefficients of the crossover exponent $\varphi_{2}$ for $n=1$. They are listed in the last column.

For the special cases where analytic results are available, we have

$$
C(2)=\frac{1}{324}
$$

and

$$
C(6)=\frac{1}{27}\left[\frac{4}{3} \ln \frac{4}{3}-\frac{19}{81}\right] .
$$

As we see, in all cases, $\varphi_{2}$ is positive at this order. Thus, we conclude that the isotropic fixed point $\mathcal{P}_{\text {iso }}^{*}$ is unstable against perturbations from cubic anisotropy of the form included in the Hamiltonian (10).

Of specific interest is the scalar biaxial case $m=2, n$ $=1$, corresponding to a biaxial generalization of the threedimensional ANNNI model discussed in the Introduction. The upper critical dimension being 5, let us set $\epsilon=2$ to consider a physical system in $d=3$ :

$$
\varphi_{2}(n=1, m=2, d=3)=\frac{1}{81} \simeq 0.0123 .
$$

Since this exponent is so small, we see that, unless the anisotropic amplitudes are large, we must be extremely close to the Lifshitz point (by careful tuning of the two control parameters) in order to detect any serious deviations from the critical behavior in the class of the isotropic fixed point $\mathcal{P}_{\text {iso }}^{*}$.

\section{CONCLUDING REMARKS}

We studied the critical properties of $m$-axial Lifshitz points in systems with spatial anisotropic interactions. Specially, for an $m$-dimensional subspace of $\mathbb{R}^{d}$ in which a wavevector instability occurs, we considered the effects of arbitrary fourth-order couplings of the form

$$
\mathcal{T}_{\alpha_{1} \alpha_{2} \alpha_{3} \alpha_{4}} q_{\alpha_{1}} q_{\alpha_{2}} q_{\alpha_{3}} q_{\alpha_{4}} \boldsymbol{\phi}_{q} \cdot \boldsymbol{\phi}_{-q},
$$

with $\boldsymbol{\phi}$ being an $n$-component order parameter. Unlike previous studies of "anisotropic" Lifshitz points, ${ }^{21}$ we are not concerned with couplings that break the $\mathrm{O}(n)$ symmetry of the order parameter. In this sense, our $\boldsymbol{\phi}$ is more appropriate for, say, multicomponent alloys than for spin systems. Especially for the $n=1$ (Ising) case, we showed how anisotropy of the form (57) naturally arises from a generalized ANNNI model. In the case we explicitly considered only a cubic anisotropy, corresponding to a tensor (8), was present [cf. Eqs. (9) and (10)].

Using field-theoretic renormalization group techniques and expanding about the upper critical dimension, we found that this is a relevant perturbation for an isotropic $m$-axial Lifshitz point. To $O\left(\epsilon^{2}\right)$, the crossover exponent has been computed for a range of $m$, with analytic forms for the special cases of $m=2,6$. Though we have not obtained similar results for general anisotropic interactions, there is no doubt that cubic anisotropy of the form included in the Hamiltonian (10) will be generically present even in systems with lower symmetries, leading to crossover in general. Since all previous investigations ${ }^{31}$ of $m$-axial Lifshitz points (with $m>1$ ) are based on $m$-isotropic Hamiltonians, our conclusion is that, unless the microscopics of a system enforces rotational invariance in $\mathbb{R}^{m}$, the critical properties will not fall into the universality classes found so far. Nevertheless, we should caution that, though the crossover exponent is positive, its numerical values are relatively small. For example, in the case most likely to be physically accessible $(d=3, m=2, n$ $=1$ ), this exponent is only $1 / 81$. Though measurable deviations from the isotropic class may be difficult to detect for real systems, it should be interesting to test our predictions in Monte Carlo simulations of suitably designed lattice models.

A more interesting question is, given the RG flow is away from the $m$-isotropic fixed point, whether there is a new stable fixed point or not. Of course, if the former is true, then we have a new universality class. However, preliminary studies indicate that the $\beta$ function for $w$ has no zero, for any finite, nonvanishing $w$. Worse, the flow seems to run indefinitely towards Hamiltonians associated with singular propagators (i.e., parts quadratic in $\boldsymbol{\phi}$ no longer being positive definite), so that $O\left(\boldsymbol{q}^{6}\right)$ terms will be needed to stabilize the theory. In addition, we might speculate that the system would undergo a first-order transition, albeit a weak one. Clearly, further investigations are necessary before definitive conclusions can be reached.

\section{ACKNOWLEDGMENTS}

We gratefully acknowledge the financial support of this work by the Alexander von Humboldt Foundation, the U.S. National Science Foundation (through Grant No. DMR0088451), and by the Deutsche Forschungsgemeinschaft (DFG) - in its initial phase via the Leibniz program (Grant No. Di 378/2) and Sonderforschungsbereich 237, and in its final phase via DFG Grant No. Di-378/3. R.K.P.Z. and M.A.Sh. thank Fachbereich Physik for their hospitality at the University of Duisburg-Essen.

\section{APPENDIX A: CALCULATION OF THE TWO-LOOP GRAPH OF $\Gamma^{(2)}$ TO ORDER $\stackrel{\circ}{\sigma}_{2}$}

Let us denote momenta in $\mathbb{R}^{d}$ by $\boldsymbol{q}=(\boldsymbol{k}, \boldsymbol{p})$ with components $\boldsymbol{k} \in \mathbb{R}^{m}$ and $\boldsymbol{p} \in \mathbb{R}^{\bar{m}}(\bar{m} \equiv d-m)$. As we will be dealing with cubic spatial anisotropy extensively, let us define

$$
\check{k}^{4} \equiv \sum_{\alpha=1}^{m} k_{\alpha}^{4},
$$

which is to be distinguished from the usual $k^{4} \equiv(\boldsymbol{k} \cdot \boldsymbol{k})^{2}$. At the Lifshitz point $\tau=\rho=0$, the Fourier transform of $G(x)$, the free propagator in position space, is 


$$
\widetilde{G}(\boldsymbol{q}) \equiv \widetilde{G}(\boldsymbol{k}, \boldsymbol{p})=\left[p^{2}+\stackrel{\circ}{\sigma}_{1} k^{4}+\stackrel{\circ}{\sigma}_{2} \check{k}^{4}\right]^{-1} .
$$

To two loops, $\widetilde{\Gamma}^{(2)}(\boldsymbol{q})$, the Fourier transform of the bare vertex function $\Gamma^{(2)}$, is given by

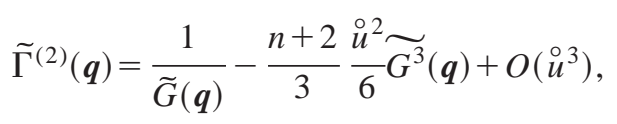

where $\widetilde{G^{3}}(\boldsymbol{q})$ denotes the Fourier transform of $G^{3}(\boldsymbol{x})$. In addition, at this order of the $\epsilon$ expansion, we will need only the residues of the simple pole of $\widetilde{\Gamma}^{(2)}$ at $\epsilon=0$. Thus, all coefficients of $\epsilon^{-1}$ may be evaluated at $\epsilon=0$, or $d=d^{*}=4$ $+m / 2$.

Let us compute the pole terms of $\widetilde{G^{3}}$ to first order in $\stackrel{\circ}{w}$ $\equiv \stackrel{\circ}{\sigma}_{2} / \stackrel{\circ}{\sigma}_{1}$. Since the dependence on $\stackrel{\circ}{\sigma}_{1}$ follows from dimensional considerations, we temporarily set $\stackrel{\circ}{\sigma}_{1}=1$. Expanding in $\stackrel{\circ}{w}$, we have

$$
\widetilde{G^{3}}=\left[\widetilde{G^{3}}\right]_{\stackrel{\circ}{w}=0}+\stackrel{\circ}{w}\left[\partial_{\dot{w}} \widetilde{G^{3}}\right]_{\stackrel{\circ}{w}=0}+O\left(\stackrel{\circ}{w^{2}}\right) .
$$

The pole contribution of the first term on the right-hand side has been computed in Ref. 12. Expressed in terms of the integrals $j_{\phi}(m)$ and $j_{\sigma}(m)$ of Ref. 13 [see its equations (43), (44), and (46)], the result is

$$
\left[\widetilde{G^{3}}\right]_{\dot{\imath}=0}=\frac{F_{m, \epsilon}^{2}}{\epsilon}\left[-\frac{j_{\phi}(m)}{2(8-m)} p^{2}+\frac{j_{\sigma}(m)}{16 m(m+2)} k^{4}\right]+O\left(\epsilon^{0}\right) .
$$

To compute the $\stackrel{\circ}{w}$ derivative appearing in Eq. (A4), we start from its position-space representation, $G^{3}(\boldsymbol{x})$, and obtain

$$
\left[\partial_{\dot{*}} G^{3}(\boldsymbol{x})\right]_{\dot{w}=0}=-3 G_{0}^{2}(\boldsymbol{x}) \sum_{\alpha} \partial_{\alpha}^{4}\left(G_{0} * G_{0}\right)(\boldsymbol{x}) .
$$

Here $\left.G_{0}(\boldsymbol{x}) \equiv G(\boldsymbol{x})\right|_{\dot{w}=0}$, and $\left(G_{0} * G_{0}\right)(\boldsymbol{x})$ means a convolution in position space (i.e., Fourier transform of $\left[\widetilde{G}_{0}(\boldsymbol{q})\right]^{2}$ ).

Next, let us exploit the scaling forms of both $G_{0}$ and $G_{0} * G_{0}$. Defining the radii

$$
r \equiv \sqrt{x_{\alpha} x_{\alpha}}, \quad R \equiv \sqrt{x_{\beta} x_{\beta}},
$$

the scaling variables

$$
v_{\alpha} \equiv x_{\alpha} R^{-1 / 2}, \quad z \equiv v_{\alpha} v_{\alpha}=v^{2},
$$

and the vectors $\boldsymbol{v} \equiv\left\{v_{\alpha}\right\}$ and $\boldsymbol{e} \equiv\left\{x_{\beta}\right\} / R$, we write

$$
G_{0}(\boldsymbol{x})=R^{-2+\epsilon} \Phi_{m, d}(v)
$$

and

$$
\left(G_{0} * G_{0}\right)(\boldsymbol{x})=R^{\epsilon} Y_{m, d}(z) .
$$

Here, the scaling functions are

$$
\Phi_{m, d}(v) \equiv \int_{k}^{(m)} \int_{p}^{(\bar{m})} \frac{e^{i \boldsymbol{k} \cdot \boldsymbol{v}+i \boldsymbol{p} \cdot \boldsymbol{e}}}{k^{4}+p^{2}}
$$

and

$$
Y_{m, d}\left(v^{2}\right) \equiv \int_{\boldsymbol{k}}^{(m)} \int_{\boldsymbol{p}}^{(\bar{m})} \frac{e^{i \boldsymbol{k} \cdot \boldsymbol{v}+i \boldsymbol{p} \cdot \boldsymbol{e}}}{\left(k^{4}+p^{2}\right)^{2}}
$$

where $\int_{\boldsymbol{k}}^{(m)} \equiv(2 \pi)^{-m} \int d^{m} k$ and $\int_{\boldsymbol{p}}^{(\bar{m})} \equiv(2 \pi)^{-\bar{m}} \int d^{\bar{m}} p$. Their explicit expressions in terms of Taylor series and hypergeometric functions, as well as the asymptotic expansions for large $z$, are given in Appendix B.

Inserting the above scaling forms into Eq. (A6) and performing the Fourier transformation, we encounter integrations over $\boldsymbol{x}$. Using hyperspherical coordinates $\left(v \sqrt{R}, \Omega_{m}\right)$ in $\mathbb{R}^{m}$ and $\left(R, \Omega_{\bar{m}}\right)$ in $\mathbb{R}^{\bar{m}}$, let us denote angular averages by

$$
\bar{f}^{D} \equiv S_{D}^{-1} \int f\left(\Omega_{D}\right) d \Omega_{D}
$$

where $S_{D} \equiv \int d \Omega_{D}=2 \pi^{D / 2} / \Gamma(D / 2)$ is the surface area of a $D$-dimensional unit sphere. In the radial integration $\int_{0}^{\infty} d R$, the distribution $R^{-3+2 \epsilon}$ is found to appear. Employing its Laurent expansion ${ }^{32}$

$$
R^{-3+2 \epsilon}=\frac{1}{4 \epsilon} \delta^{\prime \prime}(R)+O\left(\epsilon^{0}\right)
$$

we find

$$
\begin{aligned}
{\left[\partial_{\dot{\circ}} \tilde{G}^{3}\right]_{\mathcal{\circ}=0}=} & \frac{-3}{4 \epsilon} S_{\bar{m}} \frac{\partial^{2}}{\partial R^{2}} \overline{e^{i R \boldsymbol{p} \cdot \boldsymbol{e}}} \bar{m} \\
& \times\left.\sum_{\alpha} d_{v_{\alpha}}^{4} Y_{m, d *}\left(\boldsymbol{v}^{2}\right)\right|_{R=0} ^{i \sqrt{R} \boldsymbol{k} \cdot \boldsymbol{v}} \Phi_{m, d *}^{2}+O\left(\boldsymbol{\epsilon}^{0}\right)
\end{aligned}
$$

The rightmost part of Eq. (A15) can be rewritten as

$$
\begin{aligned}
\sum_{\alpha=1}^{m} \partial_{v_{\alpha}}^{4} Y_{m, d *}\left(v^{2}\right)= & 12 m Y_{m, d^{*}}^{\prime \prime}\left(v^{2}\right)+48 v^{2} Y_{m, d^{*}}^{\prime \prime \prime}\left(v^{2}\right) \\
& +16 \sum_{\alpha} v_{\alpha}^{4} Y_{m, d^{*}}^{(i v)}\left(v^{2}\right),
\end{aligned}
$$

where $Y^{(i v)}(z) \equiv d^{4} Y(z) / d z^{4}$.

Note that the function of $R$ that must be differentiated in Eq. (A15) has an expansion in even powers of $\sqrt{R}$ because the angular integration in $\int d^{m} v$ yields zero for the coefficients of all odd powers. Thus only the contributions $\propto R^{2}$ produced by the two exponential functions contribute to the pole term on the right-hand side.

For the angular averages, we may exploit the characteristic function

$$
{\overline{e^{i k \cdot v}}}^{m}=\sum_{\ell=0}^{\infty} \frac{(i k v)^{2 \ell} \Gamma(m / 2)}{4^{\ell} \ell ! \Gamma(\ell+m / 2)}
$$

to find

$$
{\overline{\alpha_{1}}} \cdots v_{\alpha_{2 N}} m / v^{2 N}=\frac{\delta_{\alpha_{1} \alpha_{2}} \cdots \delta_{\alpha_{2 N-1} \alpha_{2 N}}+\cdots}{m(m+2)(m+4) \cdots[m+2(N-1)]} .
$$


In the numerator the ellipsis $\cdots$ stands for the remaining $(2 N-1) ! !-1$ pairings of the indices. This can also be thought of as $2 N$-point function of a Gaussian theory whose propagator between two "points" $\alpha_{i}$ and $\alpha_{j}$ is given by $\delta_{\alpha_{i} \alpha_{j}}$

Utilizing these results, we easily find

$$
\begin{gathered}
\frac{\partial^{2} \overline{R^{2}} e^{i R \boldsymbol{p} \cdot \boldsymbol{e}}}{\bar{m}_{R=0}}=-\frac{p^{2}}{\bar{m}}, \\
\sum_{\alpha}{\overline{v_{\alpha}^{4}}}^{m}=\frac{3 v^{4}}{m+2}, \\
\left.\frac{\partial^{2}}{\partial R^{2}} e^{i \sqrt{R} \boldsymbol{k} \cdot \boldsymbol{v}}\right|_{R=0} ^{m}=\frac{k^{4} v^{4}}{4 m(m+2)},
\end{gathered}
$$

and

$$
\begin{aligned}
& \left.\frac{\partial^{2}}{\partial R^{2}} \sum_{\alpha}{\overline{v_{\alpha}^{4} e^{i \sqrt{R} \boldsymbol{k} \cdot \boldsymbol{v}}}}^{m}\right|_{R=0} \\
& \quad=\frac{3(m+8) k^{4}+\check{k}^{4}}{4 m(m+2)(m+4)(m+6)} v^{8} .
\end{aligned}
$$

Combined with Eq. (A15), these results yield

$$
\begin{aligned}
{\left[\partial_{w} \widetilde{G}^{3}\right]_{w=0}=} & \frac{F_{m, \epsilon}^{2}}{\epsilon}\left[\frac{18 i_{\phi}(m)}{8-m} p^{2}-\frac{9 i_{\sigma_{1}}(m)}{4 m(m+2)} k^{4}\right. \\
& \left.-\frac{24 i_{\sigma_{2}}(m)}{m(m+2)(m+4)(m+6)} \check{k}^{4}\right]+O\left(\epsilon^{0}\right),
\end{aligned}
$$

where we have introduced the integrals

$$
\begin{aligned}
i_{\phi}(m) \equiv & B_{m} \int_{0}^{\infty} d v v^{m-1} \Phi_{m, d^{*}}^{2}(v)\left[m Y_{m, d^{*}}^{\prime \prime}\left(v^{2}\right)\right. \\
& \left.+4 v^{2} Y_{m, d^{*}}^{\prime \prime \prime}\left(v^{2}\right)+\frac{4}{m+2} v^{4} Y_{m, d^{*}}^{(i v)}\left(v^{2}\right)\right]
\end{aligned}
$$

$$
\begin{aligned}
i_{\sigma_{1}}(m) \equiv & B_{m} \int_{0}^{\infty} d v v^{m+3}\left[m Y_{m, d *}^{\prime \prime}\left(v^{2}\right)+4 v^{2} Y_{m, d *}^{\prime \prime \prime}\left(v^{2}\right)\right. \\
& \left.+\frac{4(m+8) v^{4}}{(m+4)(m+6)} Y_{m, d^{*}}^{(i v)}\left(v^{2}\right)\right],
\end{aligned}
$$

and

$$
i_{\sigma_{2}}(m) \equiv B_{m} \int_{0}^{\infty} d v v^{m+7} \Phi_{m, d^{*}}^{2}(v) Y_{m, d^{*}}^{(i v)}\left(v^{2}\right)
$$

with

$$
B_{m} \equiv \frac{S_{4-m / 2} S_{m}}{F_{m, 0}^{2}}=\frac{2^{10+m} \pi^{6+3 m / 4} \Gamma(m / 2)}{\Gamma(2-m / 4) \Gamma^{2}(m / 4)} .
$$

For completeness, we recall the definitions ${ }^{13}$ of

$$
j_{\phi}(m) \equiv B_{m} \int_{0}^{\infty} d v v^{m-1} \Phi_{m, d^{*}}^{3}(v)
$$

and

$$
j_{\sigma}(m) \equiv B_{m} \int_{0}^{\infty} d v v^{m+3} \Phi_{m, d^{*}}^{3}(v) .
$$

\section{APPENDIX B: THE SCALING FUNCTIONS $\Phi_{m, d}(v)$ AND $Y_{m, d}(z)$}

First, let us recall the properties of the scaling function $\Phi_{m, d}(v)$, which were established in Refs. 12 and13. Its Taylor expansion is

$$
\Phi_{m, d}(v)=\frac{\pi^{(1-d) / 2}}{2^{2+m}} \sum_{\ell=0}^{\infty} \frac{\Gamma(\ell / 2+1-\epsilon / 2)}{\ell ! \Gamma\left(\ell / 2+\frac{1}{2}+m / 4\right)}\left(-\frac{v^{2}}{4}\right)^{\ell}
$$

It is possible to express the power series in closed form by exploiting a relation of the generalized hypergeometric functions ${ }_{1} F_{2}$ (by summing the contributions with even and odd values of $\ell$ separately), namely,

$$
\begin{aligned}
\sum_{\ell=0}^{\infty} \frac{1}{\ell !} \frac{\Gamma(a+\ell / 2)}{\Gamma(b+\ell / 2)}(-y)^{\ell} \\
=\frac{\Gamma(a)}{\Gamma(b)}{ }_{1} F_{2}\left(a ; \frac{1}{2}, b ; \frac{y^{2}}{4}\right) \\
\quad-y \frac{\Gamma\left(a+\frac{1}{2}\right)}{\Gamma\left(b+\frac{1}{2}\right)}{ }_{1} F_{2}\left(a+\frac{1}{2} ; \frac{3}{2}, b+\frac{1}{2} ; \frac{y^{2}}{4}\right) .
\end{aligned}
$$

The large $v$ properties of ${ }_{1} F_{2}$ lead us to the asymptotic expansion

$$
\Phi_{m, d^{*}}(v) \underset{v \rightarrow \infty}{\approx} \frac{2^{1-m}}{\pi^{(6+m) / 4}} \frac{m-2}{\Gamma\left(\frac{1}{2}+m / 4\right)} v^{-4}\left[1+O\left(v^{-4}\right)\right]
$$

which will be used in numerical evaluations of $i_{\sigma_{2}}(m)$ described in Sec. III.

The scaling function $Y_{m, d}\left(v^{2}\right)$ was also studied in Ref. 13: It appeared in the calculation of another scaling function, $\Theta(v)$, that was needed for the coupling constant renormalization at the two-loop level. Its Taylor expansion, from Eq. (D4) in this reference, reads 


$$
Y_{m, d}(z)=\frac{\pi^{(1-d) / 2}}{2^{4+m}} \sum_{\ell=0}^{\infty} \frac{\Gamma(\ell / 2-\epsilon / 2)}{\ell ! \Gamma\left(\ell / 2+\frac{1}{2}+m / 4\right)}\left(-\frac{z}{4}\right)^{\ell} .
$$

As for $\Phi$ above, we can write a closed form for this function in terms of two hypergeometric functions:

$$
\begin{aligned}
Y_{m, d}(z)= & \frac{\pi^{(1-d) / 2}}{2^{4+m}}\left[\frac{\Gamma(-\epsilon / 2)}{\Gamma\left(\frac{1}{2}+m / 4\right)}{ }_{1} F_{2}\left(-\frac{\epsilon}{2} ; \frac{1}{2}, \frac{1}{2}+\frac{m}{4} ; \frac{z^{2}}{64}\right)\right. \\
& \left.-\frac{z}{4} \frac{\Gamma\left(\frac{1}{2}-\epsilon / 2\right)}{\Gamma(1+m / 4)}{ }_{1} F_{2}\left(\frac{1}{2}-\frac{\epsilon}{2} ; \frac{3}{2}, 1+\frac{m}{4} ; \frac{z^{2}}{64}\right)\right] .
\end{aligned}
$$

Let us note that, though the series (B4) starts with a term that has a pole in $\epsilon$, all its derivatives are regular at $\epsilon=0$. In particular, for the calculation of the integral $i_{\sigma_{2}}(m)$, we need only $Y_{m, d^{*}}^{(i v)}(z)$ at the upper critical dimension $d^{*}=4+m / 2$. Thus,

$$
Y_{m, d^{*}}^{(i v)}(z)=\frac{1}{2^{12+m} \pi^{(6+m) / 4}} \sum_{\ell=0}^{\infty} \frac{1}{\ell !} \frac{\Gamma(2+\ell / 2)(-z / 4)^{\ell}}{\Gamma\left(\frac{5}{2}+m / 4+\ell / 2\right)}
$$

$$
\begin{aligned}
= & \frac{1}{2^{14+m} \pi^{(6+m) / 4}}\left[\frac{4}{\Gamma\left(\frac{5}{2}+m / 4\right)}{ }_{1} F_{2}\left(2 ; \frac{1}{2}, \frac{5}{2}+\frac{m}{4} ; \frac{z^{2}}{64}\right)\right. \\
& \left.-\frac{z \Gamma\left(\frac{5}{2}\right)}{\Gamma(3+m / 4)}{ }_{1} F_{2}\left(\frac{5}{2} ; \frac{3}{2}, 3+\frac{m}{4} ; \frac{z^{2}}{64}\right)\right] .
\end{aligned}
$$

In the special cases $m=2$ and $m=6$, the hypergeometric functions here reduce to the elementary functions:

$$
Y_{2,5}^{(i v)}(z)=\frac{3}{(4 \pi)^{2} z^{4}}\left[1-e^{-z / 4}\left(1+\frac{z}{4}+\frac{z^{2}}{32}+\frac{z^{3}}{384}\right)\right]
$$

and

$$
Y_{6,7}^{(i v)}(z)=\frac{48\left(-320+z^{2}\right)+e^{-z / 4}\left[15360+z(16+z)\left(240+12 z+z^{2}\right)\right]}{(4 \pi)^{3} 64 z^{6}} .
$$

For the integral $i_{\sigma_{2}}(m)$, we will need the asymptotic expansion of $Y_{m, d^{*}}^{(i v)}(z)$ :

$$
Y_{m, d^{*}}^{(i v)}(z) \underset{z \rightarrow \infty}{\approx} \frac{3}{2^{2+m} \pi^{(6+m) / 4} \Gamma\left(\frac{1}{2}+\frac{m}{4}\right)} z^{-4}\left[1+O\left(z^{-2}\right)\right] .
$$

Let us, however, note that, for arbitrary values of $m$, derivatives of $Y_{m, d *}(z)$ can be expressed completely in terms of $\Phi_{m, d^{*}}(v)$ (with $\left.v=\sqrt{z}\right),{ }^{12,13}$ since their Taylor series expansions differ only slightly. In particular, using

$$
\Gamma\left(\frac{\ell}{2}+1-\frac{\epsilon}{2}\right)=\left(\frac{\ell}{2}-\frac{\epsilon}{2}\right) \Gamma\left(\frac{\ell}{2}-\frac{\epsilon}{2}\right)
$$

we get the general relation

$$
\Phi_{m, d}(\sqrt{z})=2\left[z Y_{m, d}^{\prime}(z)-\epsilon Y_{m, d}(z)\right] .
$$

Recalling that $Y_{m, d}$ has a pole at $\epsilon=0$, we verify that $Y_{m, d}(z)=-\Phi_{m, d}(0) /(2 \epsilon)+O\left(\epsilon^{0}\right)$. Thus, in the limit $\epsilon$ $\rightarrow 0$, we find an exceedingly simple relationship between $Y_{m, d}^{\prime}$ and $\Phi_{m, d}$ :

$$
Y_{m, d *}^{\prime}(z)=\frac{1}{2 z}\left[\Phi_{m, d *}(\sqrt{z})-\Phi_{m, d^{*}}(0)\right] .
$$

In other words, the scaling function $\Phi_{m, d^{*}}$ completely determines all derivatives of $Y_{m, d^{*}}$, a fact we will exploit in the following appendix.

\section{APPENDIX C: ANALYTIC RESULTS FOR $m=2$ AND $m=6$}

The special case $m=2$ is the simplest one since the scaling function $\Phi$ acquires an extremely simple form at the upper critical dimension:

$$
\Phi_{2,5}(\sqrt{z})=\frac{1}{(4 \pi)^{2}} e^{-z / 4} .
$$

For the function $Y_{2,5}^{\prime}$, using the relation (B13), we thus get

$$
Y_{2,5}^{\prime}(z)=\frac{1}{(4 \pi)^{2}} \frac{1}{2 z}\left(e^{-z / 4}-1\right) .
$$

Even more simplifications of integrals are realized if we use the representation 


$$
\int_{0}^{1} d t e^{-z t / 4}=\frac{4}{z}\left(1-e^{-z / 4}\right)
$$

so that

$$
Y_{2,5}^{(i v)}(z)=\frac{1}{(4 \pi)^{2}} \frac{2}{4^{5}} \int_{0}^{1} d t t^{3} e^{-z t / 4} .
$$

Inserting this expression into Eq. (A26) for the integral $i_{\sigma_{2}}$, we find

$$
i_{\sigma_{2}}(2)=2 \int_{0}^{\infty} d \zeta \zeta^{4} e^{-2 \zeta} \int_{0}^{1} d t t^{3} e^{-\zeta t}
$$

To arrive here, we changed the integration variable to $\zeta$ $\equiv z / 4$ for convenience. Carrying out the trivial integrations, we get

$$
i_{\sigma_{2}}(2)=2 \times 4 ! \int_{0}^{1} d t t^{3}(t+2)^{-5}=\frac{2}{27} \text {. }
$$

The special case $m=6$ is, as usual, somewhat more involved. This is due to a more complicated functional form of the corresponding scaling function $\Phi$. Using $\zeta=z / 4$ once more, we have

$$
\Phi_{6,7}(\sqrt{4 \zeta})=\frac{1}{2(4 \pi)^{3}} \frac{1}{\zeta^{2}}\left[1-(1+\zeta) e^{-\zeta}\right] .
$$

Again, we exploit an integral representation like Eq. (C3) now, for both functions $\Phi_{6,7}$ and $Y_{6,7}^{\prime}$. Thus, Eq. (C7) can be written as

$$
\Phi_{6,7}(\sqrt{4 \zeta})=\frac{1}{2(4 \pi)^{3}} \int_{0}^{1} d t t e^{-\zeta t} .
$$

Inserting this into the relation (B13), we obtain

$$
Y_{6,7}^{\prime}(z)=-\frac{1}{16(4 \pi)^{3}} \int_{0}^{1} d t t^{2} \int_{0}^{1} d y e^{-z t y / 4}
$$

and

$$
Y_{6,7}^{(i v)}(z)=\frac{1}{4^{5}(4 \pi)^{3}} \int_{0}^{1} d t t^{5} \int_{0}^{1} d y y^{3} e^{-z t y / 4} .
$$

The desired integral $i_{\sigma_{2}}(6)$ thus becomes

$$
\begin{aligned}
i_{\sigma_{2}}(6)= & 4 \int_{0}^{\infty} d \zeta \zeta^{6} \int_{0}^{1} d \alpha \alpha e^{-\alpha \zeta} \int_{0}^{1} d \beta \beta e^{-\beta \zeta} \\
& \times \int_{0}^{1} d t t^{5} \int_{0}^{1} d y y^{3} e^{-\zeta t y} \\
= & 4 \times 6 ! \int_{0}^{1} d \alpha \alpha \int_{0}^{1} d \beta \beta \int_{0}^{1} d t t^{5} \int_{0}^{1} d y y^{3} \\
& \times \frac{1}{(\alpha+\beta+t y)^{7}} \\
= & 20\left(8 \ln \frac{4}{3}-\frac{59}{27}\right) .
\end{aligned}
$$

The integration over $\zeta$ was trivial, and the last result was obtained by simple repeated integrations with the help of MATHEMATICA. $^{29}$
${ }^{1}$ M. E. Fisher, in Critical Phenomena, edited by F. J. W. Hahne, Vol. 186 of Lecture Notes in Physics (Springer-Verlag, Berlin, 1983), pp. 1-139.

${ }^{2}$ M.E. Fisher, Rev. Mod. Phys. 70, 653 (1998).

${ }^{3}$ A. Aharony, in Phase Transitions and Critical Phenomena, edited by C. Domb and J. L. Lebowitz (Academic, London, 1976), Vol. 6, pp. 358-424.

${ }^{4}$ W. Selke, in Phase Transitions and Critical Phenomena, edited by C. Domb and J. L. Lebowitz (Academic, London, 1992), Vol. 15 , pp. $1-72$.

${ }^{5}$ M.E. Fisher and W. Selke, Phys. Rev. Lett. 44, 1502 (1980).

${ }^{6}$ R.M. Hornreich, M. Luban, and S. Shtrikman, Phys. Rev. Lett. 35, 1678 (1975).

${ }^{7}$ For background and extensive lists of references, see the review articles in Refs. 8 and 9.

${ }^{8}$ R.M. Hornreich, J. Magn. Magn. Mater. 15-18, 387 (1980).

${ }^{9}$ H.W. Diehl, Acta Phys. Slov. 52, 271 (2002), Proceedings of the 5th International Conference "Renormalization Group 2002," Tatranska Strba, High Tatra Mountains, Slovakia, March 2002.

${ }^{10}$ C. Mergulhão, Jr. and C.E.I. Carneiro, Phys. Rev. B 58, 6047 (1998).

${ }^{11}$ C. Mergulhão, Jr. and C.E.I. Carneiro, Phys. Rev. B 59, 13954 (1999)
${ }^{12}$ H.W. Diehl and M. Shpot, Phys. Rev. B 62, 12338 (2000).

${ }^{13}$ M. Shpot and H.W. Diehl, Nucl. Phys. B 612, 340 (2001).

${ }^{14}$ H.W. Diehl and M. Shpot, J. Phys. A 34, 9101 (2001).

${ }^{15}$ H.W. Diehl and M. Shpot, J. Phys. A 35, 6249 (2002).

${ }^{16}$ J. Sak and G.S. Grest, Phys. Rev. B 17, 3602 (1978).

${ }^{17}$ R.M. Hornreich and A.D. Bruce, J. Phys. A 11, 595 (1978).

${ }^{18}$ D. Mukamel, J. Phys. A 10, L249 (1977).

${ }^{19}$ M.M. Leite, Phys. Rev. B 67, 104415 (2003).

${ }^{20}$ H.W. Diehl and M. Shpot, Phys. Rev. B 68, 066401 (2003).

${ }^{21}$ R.M. Hornreich, Phys. Rev. B 19, 5914 (1979).

${ }^{22}$ R. Folk and G. Moser, Phys. Rev. B 47, 13992 (1993).

${ }^{23}$ L. D. Landau and E. M. Lifschitz, Elastizitätstheorie, 5th ed., Vol. VII of Lehrbuch der Theoretischen Physik (Akademie Verlag, Berlin, 1983).

${ }^{24}$ There are fewer tensors $\mathcal{T}_{\alpha_{1} \alpha_{2} \alpha_{3} \alpha_{4}}$ than elastic moduli $C_{\alpha_{1} \alpha_{2} ; \alpha_{3} \alpha_{4}}$ because the latter are not totally symmetric but satisfy in general only the relations (Ref. 23) $C_{\alpha_{1} \alpha_{2} ; \alpha_{3} \alpha_{4}}=C_{\alpha_{2} \alpha_{1} ; \alpha_{3} \alpha_{4}}$ $=C_{\alpha_{1} \alpha_{2} ; \alpha_{4} \alpha_{3}}=C_{\alpha_{3} \alpha_{4} ; \alpha_{1} \alpha_{2}}$.

${ }^{25}$ To investigate the Lifshitz-point critical behavior in the spherical limit $n \rightarrow \infty$, Frachebourg and Henkel (Ref. 26) considered a Hamiltonian of the form (10) but without the isotropic derivative term $\propto \stackrel{\circ}{\sigma}_{1}$. In RG analyses such a simplifying assumption cannot 
be maintained because this term gets generated under the RG, even within the framework of the $1 / n$ expansion.

${ }^{26}$ L. Frachebourg and M. Henkel, Physica A 195, 577 (1993).

${ }^{27}$ H.W. Diehl, A. Gerwinski, and S. Rutkevich, cond-mat/0308483 (unpublished).

${ }^{28}$ F. Wegner, Phys. Rev. B 5, 4529 (1972).

${ }^{29}$ MATHEMATICA, version 3.0, a product of Wolfram Research.

${ }^{30}$ We have verified that our numerical integration method gives values for $m=2$ and $m=6$ in conformity with the analytical results (52). Yet, in the $m=6$ case, we have contented ourselves with a numerical accuracy $\leqslant 0.4 \%$. The additional decimals given in Table I were determined from the exact expression (52). For $m=2$, the numerical accuracy is higher, corresponding essentially to the number of decimals given.

${ }^{31}$ The sole exception, to our knowledge, is D. Mukamel and M. Luban, Phys. Rev. B 18, 3631 (1978).

${ }^{32}$ I. M. Gel'fand and G. E. Shilov, Generalized Functions (Academic, New York, 1964), Vol. 1. 